\title{
Response to Letter to the Editor Regarding "Abiraterone Acetate for the Treatment of Chemotherapy-Naïve Metastatic Castration- Resistant Prostate Cancer: An Evidence Review Group Perspective of a NICE Single Technology Appraisal"
}

\author{
Bram L. T. Ramaekers ${ }^{1}$ (D) Rob Riemsma $^{2} \cdot$ Florian Tomini $^{1} \cdot$ Thea van Asselt $^{1,3,4}$. \\ Sohan Deshpande ${ }^{2} \cdot$ Steven Duffy $^{2} \cdot$ Nigel Armstrong $^{2} \cdot$ Johan L. Severens $^{5} \cdot$ \\ Jos Kleijnen $^{2,6} \cdot$ Manuela A. Joore ${ }^{1}$
}

Published online: 8 April 2017

(C) Springer International Publishing Switzerland 2017

Dear Editors,

We very much appreciate the interest Reifsnider et al. [1] have taken in our perspective on the single technology appraisal (STA) of abiraterone acetate (tradename Zytiga ${ }^{\circledR}$ ) plus prednisolone (AAP) acetate for the treatment of chemotherapynaive metastatic castration-resistant prostate cancer (mCRPC) prepared for the UK National Institute for Health and Care Excellence (NICE) [2, 3]. Here, we respond to the aspects highlighted by Reifsnider et al. [1] and correct factual inaccuracies in their reasoning. The main issues are printed in italics [1]. These points relate to (1) the statistical significance of the COU-AA-302 survival results and (2) the justification of the

This is a reply to the letter available at doi:10.1007/s40273-017-0502-8.

Bram L. T. Ramaekers

bram.ramaekers@mumc.nl

1 Department of Clinical Epidemiology and Medical Technology Assessment (KEMTA), Maastricht University Medical Center, Maastricht, The Netherlands

2 Kleijnen Systematic Reviews Ltd, York, UK

3 Department of Epidemiology, University Medical Center Groningen, University of Groningen, Groningen, The Netherlands

4 Department of Pharmacy, University of Groningen, Groningen, The Netherlands

5 Institute of Health Policy and Management, iMTA-Institute for Medical Technology Assessment, Erasmus University Rotterdam, Rotterdam, The Netherlands

6 School for Public Health and Primary Care (CAPHRI), Maastricht University, Maastricht, The Netherlands discrete-event simulation (DES) approach utilised by the company. Reifsnider et al. [1] also highlight aspects that are unrelated to these main points, and we discuss these separately.

\section{Statistical Significance of the COU-AA-302 Survival Results}

1. The ERG [evidence review group] alleged that "it is unlikely that the trial will ever show a significant survival benefit"; however, the final COU-AA-302 overall survival $(O S)$ results were statistically significant regardless of crossover [1].

We stated that it was unlikely that the trial would ever show a significant survival benefit based on the original unadjusted analysis [2, 3]. The results reported by Ryan et al. [4] are adjusted for cross-over. These results were published in January 2015, 10 months after publication of the final Evidence Review Group (ERG) report in March 2014. In addition, the NICE appraisal committee (AC) criticized the conduct of the trial by stating,

"The committee was aware that the company unblinded COU-AA-302 early between the second and third interim analyses for overall survival and that, at both of these interim analyses, the results for overall survival did not show a statistically significant difference between the treatment arms according to the pre-specified statistical significance levels." [5]

"The committee discussed the potential effects of stopping the trial early on the size of the estimates of 
overall survival. The committee noted a systematic review published in 2010 (Bassler et al.) [6] describing the bias in trials that stop early for benefit. Specifically, compared with trials that run to completion, trials that stop early for benefit overestimate the magnitude of the treatment effect (that is, have pooled hazard ratios around $30 \%$ lower than trials that run to completion). The company expressed the belief during consultation that this bias did not apply to oncology trials, because the review by Bassler et al. [6] included a minority of haematology/oncology trials, and because other studies indicate only a marginal bias towards overestimation in oncology trials that stopped early. However, the committee concluded that this bias was unrelated to disease area." [5]

\section{Justification of the Discrete-Event Simulation Approach Utilised}

The following points highlighted by Reifsnider et al. [1] consider the justification of the DES approach utilised:

2. The model was designed to simulate the mCRPC patient pathway whilst capturing outcomes from COU-AA-302 [1].

3. A DES model utilizing a series of prediction equations to estimate time-to-event more directly incorporated trial data, captured patient and treatment interdependencies, and improved modelling efficiency [1].

4. Whilst the ERG heavily criticized the DES approach, the NICE Appraisal Committee ultimately accepted the approach and recommended AAP within its marketing authorization [1].

We would like to point out here that we did not criticize the DES approach itself but rather the implementation of the DES approach, as we do not believe that this was:

"the most transparent approach possible to address the decision problem defined in the scope. Transparency is a key aspect of modelling and in this specific case a more transparent model would be more convenient for an external reviewer to assess face validity and internal validity of the model." [2]

As mentioned in our original publication, the $\mathrm{AC}$ seemed to agree with us:

"The committee noted that, for two equations, the company had not followed its own statistical plan when choosing covariates, and the committee agreed that this could introduce bias to the model." [2,7]

“The committee concluded that the company's model was complex and lacked transparency, which made it difficult for the ERG to validate and critique, and for the AC to determine the plausibility of the model outcomes." [2, 7]

This indicates that, although the $\mathrm{AC}$ based their decision on the DES model utilised by the company, this does not imply that the AC also supported the DES approach as implemented by the company. In this specific case, an individual patient-level state transition (i.e. Markov) model might have been easier to implement in a transparent fashion (as highlighted in the original ERG report [3]).

\section{Aspects that are Unrelated to the Main Points}

Reifsnider et al. [1] considered it appropriate to use the complete case analysis instead of the intention-to-treat (ITT) population (excluding 187 patients [17\%] with incomplete baseline information). As stated previously [3], we believe that this approach would introduce bias in favour of AAP for time to treatment discontinuation (TTD) and hence OS (as OS is dependent on TTD in the DES model). This is illustrated in Figure 3 in the company's response to NICE's request for additional information [8] and the company's response to clarification letter question B4a [9]. As stated previously, we would ideally have preferred an approach that used the ITT population while imputing any missing data [3].

Finally, Reifsnider et al. [1] stated that the ERG preferred a Weibull distribution (instead of the Log-logistic distribution) to estimate TTD for the initial treatment. This is factually incorrect; we did not replace the Log-logistic distribution with the Weibull distribution in our base case (this was only explored in scenario analyses) [3].

\section{Compliance with Ethical Standards}

Funding This project was funded by the National Institute for Health Research (NIHR) Health Technology Assessment (HTA) Programme. See the HTA programme website for further project information (http://www.hta.ac.uk). The views and opinions expressed herein are those of the authors and do not necessarily reflect those of NICE or the Department of Health.

Conflicts of interest Bram Ramaekers, Rob Riemsma, Florian Tomini, Thea van Asselt, Sohan Deshpande, Steven Duffy, Nigel Armstrong, Johan Severens, Jos Kleijnen and Manuela A. Joore have no conflicts of interest.

\section{References}

1. Reifsnider O, Hall F, Sorensen S, Proskorovsky I, Girod I, Lee J. Comment on "Abiraterone acetate for the treatment of chemotherapy-naïve metastatic castration-resistant prostate cancer: an Evidence Review Group perspective of an NICE Single Technology 
Appraisal". Letter to the Editor. Pharmacoeconomics. 2017. doi:10.1007/s40273-017-0502-8.

2. Ramaekers BL, Riemsma R, Tomini F, van Asselt T, Deshpande S, Duffy $\mathrm{S}$, et al. Abiraterone acetate for the treatment of chemotherapy-naïve metastatic castration-resistant prostate cancer: an Evidence Review Group perspective of an NICE Single Technology Appraisal. Pharmacoeconomics. 2017;35(2):191-202. doi:10. 1007/s40273-016-0445-5.

3. Riemsma R, Ramaekers BLT, Tomini F, Wolff R, van Asselt ADI, Joore MA, et al. Abiraterone for the treatment of chemotherapy naïve metastatic castration-resistant prostate cancer: a Single Technology Appraisal. York: Kleijnen Systematic Reviews Ltd.; 2014. https:// www.nice.org.uk/guidance/ta387/documents/prostate-cancermetastatic-hormone-relapsed-not-treated-with-chemotherapyabiraterone-acetate-with-prednisolone-id503-evaluation-report. Accessed 21 March 2016.

4. Ryan CJ, Smith MR, Fizazi K, Saad F, Mulders PF, Sternberg CN, et al. Abiraterone acetate plus prednisone versus placebo plus prednisone in chemotherapy-naive men with metastatic castrationresistant prostate cancer (COU-AA-302): final overall survival analysis of a randomised, double-blind, placebo-controlled phase 3 study. Lancet Oncol. 2015;16(2):152-60. doi:10.1016/S14702045(14)71205-7.

5. National Institute for Health and Care Excellence (NICE). Abiraterone for treating metastatic hormone-relapsed prostate cancer not previously treated with chemotherapy. NICE final appraisal determination. London: NICE; 2014. https://www.nice. org.uk/guidance/indevelopment/ta387/documents. Accessed 21 March 2016.

6. Bassler D, Briel M, Montori VM, Lane M, Glasziou P, Zhou Q, et al. Stopping randomized trials early for benefit and estimation of treatment effects: systematic review and meta-regression analysis. JAMA. 2010;303(12):1180-7. doi:10.1001/jama.2010.310.

7. National Institute for Health and Care Excellence (NICE). Abiraterone for treating metastatic hormone-relapsed prostate cancer not previously treated with chemotherapy. NICE final appraisal determination 2. London: NICE; 2016. https://www.nice. org.uk/guidance/indevelopment/ta387/documents. Accessed 21 March 2016.

8. Janssen. Abiraterone acetate for the treatment of metastatic hormone relapsed prostate cancer not previously treated with chemotherapy [ID503]. Response to NICE request for additional information on 22 October 2015: Janssen; 2015. https://www.nice. org.uk/guidance/GID-TAG434/documents/appraisal-consultationdocument-2. Accessed 21 March 2016.

9. National Institute for Health and Care Excellence (NICE). Abiraterone acetate for the treatment of metastatic hormone relapsed prostate cancer not previously treated with chemotherapy [ID503]. Clarification letter. Manchester: NICE; 2014. https:// www.nice.org.uk/guidance/ta387/documents/prostate-cancermetastatic-hormone-relapsed-not-treated-with-chemotherapyabiraterone-acetate-with-prednisolone-id503-evaluation-report. Accessed 21 March 2016. 